\title{
Functional activities of neutrophils in diabetic rats are changed by yacon extracts
}

\section{ABSTRACT}

Introduction. Characterization of the polymorphonuclear neutrophils functional activity during the diabetes is of outmost importance for the understanding of the immunological processes involved in disease pathogenesis. The search for effective drugs that would suppress and/or modulate the immune response, preferably without negative effects, is a promising area of modern research. Smallanthus sonchifolius Poepp. et Endl., which has long been used in folk medicine to treat diabetes, was proposed as a potential treatment for immune disorders. The study aimed to search for the effective drug which would be able to regulate neutrophils functional activity in experimentally induced type 1 diabetes mellitus.

Material and methods. The experiments were conducted on male Wistar rats. Diabetes was induced by intraabdominal injection of streptozotocin. For the evaluation of phagocytic activity and the content of cationic proteins and myeloperoxidase in neutrophils cytochemical studies were performed.

Results. Diabetes development was accompanied by reduced phagocytic activity of granulocytes and increased activity of myeloperoxidase. All used extracts intensified phagocytic activity of neutrophil in diabetes, with the yacon root tubers extract demonstrating the strongest effect, which was reflected by rapid degradation of particles engulfed by phagocytes.

\footnotetext{
Address for correspondence:

Mariia Nagalievska, PhD

Department of Biochemistry

Faculty of Biology

Ivan Franko National University of Lviv

4, Hrushevskyi St., Lviv 79005, Ukraine

Phone: 00380676963530

e-mail: khmarija@gmail.com

Clinical Diabetology 2019, 8, 5, 248-253

DOI: 10.5603/DK.2019.0023

Received: 2.04.2019 Accepted: 17.07.2019
}

Conclusions. It was established that yacon extracts inhibit myeloperoxidase activity in diabetes. Therefore all investigated extracts have a pronounced immunocorrective effect and can become the basis for creating a new generation of antidiabetic drugs. (Clin Diabetol 2019; 8, 5: 248-253)

Key words: yacon, leukocytes, phagocytosis, myeloperoxidase, diabetes mellitus

\section{Introduction}

Polymorphonuclear neutrophil (PMN) is directly involved in pathogenesis of diabetes-associated complications. Neutrophils are characterized by decreased phagocytic and bactericidal activities, which lead to an increased susceptibility to infections in patients with diabetes mellitus type 1 (DM) [1]. Among these antimicrobial agents, myeloperoxidase (MPO) is the most abundant and constitutes 5\% dry weight of neutrophils and $25 \%$ of azurophilic granular proteins [2]. Accept MPO, several other proteins or enzymes are present in neutrophils which also show antimicrobial properties: defensins (major antimicrobial cationic proteins), serine proteases, NADPH oxidase, etc. [3]. Yacon (Smallanthus sonchifolius Poepp. et Endl.) has long been used in folk medicine to treat diabetes. Active components from this plant are able to stimulate immune defense by promoting antioxidant, antimicrobial, and anticancer effects [4]. This study aimed to search for the most effective yacon extract that would be able to regulate neutrophils functional activity under the experimental DM.

\section{Material and methods \\ Preparation of water extracts of yacon}

Preparation of water extracts of yacon was performed according to Nagalievska et al. [5]. For the research water solutions of evaporated extracts were used. 


\section{Induction of diabetes}

Following an overnight starvation, diabetes was induced by intraabdominal injection of streptozotocin (STZ, Sigma, USA) (freshly dissolved in $10 \mathrm{mM}$ citrate buffer [pH 5.5]) at a dose of $0.055 \mathrm{~g} / \mathrm{kg}$ body weight.

\section{Experimental animals}

Experiments were conducted using male Wistar rats weighing 150 to $220 \mathrm{~g}$. The rats were kept in the animal house and fed with a standard laboratory diet and water ad libitum. Study was carried out according to the "General ethical principles of experiments on animals" (Kyiv, 2001) and the European Convention (Strasbourg, 1986). Animals were randomly divided into following groups ( $n=5-8 /$ group): control animals (C); control animals that were treated with extract of yacon leaves $\left(C+Y_{L}\right)$; control animals that were treated with extract of yacon root tubers $\left(C+Y_{R}\right)$; animals with $D M$ (D); animals with DM that were treated with extract of yacon leaves $\left(D+Y_{L}\right)$; animals with $D M$ that were treated with extract of yacon root tubers $\left(D+Y_{R}\right)$. In the $D+Y_{L}$ and $D+Y_{R^{\prime}}$ groups the studied extracts started to be administered on the $14^{\text {th }}$ day after induction of diabetes. Groups $C+Y_{L^{\prime}} D+Y_{L^{\prime}} C+Y_{R}$ and $D+Y_{R}$ were treated by extracts at dose $70 \mathrm{mg} / \mathrm{kg}$ per day orally through a tube with preservation of precise dosage for 2 weeks.

\section{Collection of blood and leukocytes separation}

At the end of experimental period, the rats were starved for $15 \mathrm{~h}$ and then anesthetized using deep ether anesthesia method. Whole blood was collected and immediately transferred to heparinized tubes. Leukocytes were separated on gradient of Histopaque ${ }^{\circledR}$ 1083 (density of $1.083 \mathrm{~g} / \mathrm{mL}$ ).

\section{Cytochemical studies}

Phagocytic Activity Assay was conducted according to Levinsky et. al [6]. Based on obtained results next phagocytosis indexes were calculated: $\mathrm{PI}$ - phagocytic index - percentage of cells started the phagocytosis, of the total number of cells $\left(\mathrm{Pl}_{30}\right.$ and $\mathrm{Pl}_{120}$ after $30 \mathrm{~min}$ and 120 min of incubation, respectively); PN - phagocytic number - average number of yeasts cells that were inside phagocytes $\left(\mathrm{PN}_{30}\right.$ and $\mathrm{PN}_{120}$ respectively after $30 \mathrm{~min}$ and $120 \mathrm{~min}$ of incubation); IPC - index of phagocytosis completeness - division quotient of $\mathrm{PN}_{30}$ on $\mathrm{PN}_{120}$. Determination of cationic proteins content was made according to Shubych [7]. Determination of MPO level was performed by Graham-Knol method [8].

\section{Evaluation of cytochemical studies}

Evaluation of cytochemical studies were performed by semiquantitative method using Astraldi principle that is based on the differentiation of a specific color varying intensity $(0,+,++,+++)$. The results were expressed as the average cytochemical coefficient (ACC) - that was calculated using the formula:

$$
A C C=\frac{A \times 0+B \times 1+C \times 2+D \times 3}{n}
$$

where a number of cells with $A$ - negative reaction, $\mathrm{B}-$ poorly positive reaction, $\mathrm{C}-$ moderately positive reaction, $\mathrm{D}-$ sharply positive reaction, $\mathrm{n}-$ a number of counted cells.

\section{Statistical analysis}

Quantitative data obtained from the study was performed by arithmetic mean $-M$, the standard deviation of the arithmetic mean $-m$. Reliability of the difference between statistical characteristics of two alternative data sets was performed by one-way analysis of variance. The difference was considered significant if $\mathrm{P}<0.05$.

\section{Results}

In the case of DM we have established the decrease in $\mathrm{Pl}_{30}$ by $16.5 \%$ and $\mathrm{PN}_{30}$ by $19.3 \%$ compared to control. A slightly different picture was observed in the case of a longer incubation of neutrophils with yeast cells, in particular it was established the increase in $\mathrm{Pl}_{120}$ at $28.8 \%$, compared with the control. In animals with DM the increase in average number of yeasts cells inside phagocytes was observed (Table 1). The decrease in IPC in DM by $19.7 \%$ was shown compared to control value (Figure 1).

$Y_{L}$ extract administration to animals with DM resulted in 2.1-fold increase in $\mathrm{Pl}_{30}$ and $\mathrm{Pl}_{120}$ growth both in control animals and in animals with DM, respectively at $97.8 \%$ and at $75.1 \%$ compared to control. Administration of $Y_{L}$ extract to animals with DM leads to growth of $\mathrm{PN}_{30}(39.4 \%)$ and $\mathrm{PN}_{120}(8.2 \%)$ compared with diabetes. A similar growth of $\mathrm{PN}_{120}(20.9 \%)$ has been demonstrated when the extract was administrated to healthy animals (Table 1). We have established the increase in IPC after $Y_{L}$ extract treatment compared to diabetic animals (Figure 1).

Using $Y_{R}$ extract in the control animals cause $20.3 \%$ reduction of $\mathrm{Pl}_{30}$, with subsequent growth of neutrophils that entered the phagocytosis at $120 \mathrm{~min}$ (Table 1).

In DM animals, the administration of $Y_{R}$ extract caused the $\mathrm{Pl}_{30}$ increase at $50.0 \%$, compared with untreated DM animals. In the later stages of the phago- 
Table 1. Neutrophils phagocytic activity indexes under administration of yacon extracts

\begin{tabular}{|c|c|c|c|c|}
\hline & \multicolumn{2}{|c|}{ (\%) } & \multicolumn{2}{|c|}{ Number of yeast cells } \\
\hline & $\mathrm{PI}_{30}$ & $\mathrm{PI}_{120}$ & $\mathrm{PN}_{30}$ & $\mathrm{PN}_{120}$ \\
\hline C & $13.17 \pm 0.73$ & $14.75 \pm 0.88$ & $1.76 \pm 0.03$ & $1.53 \pm 0.09$ \\
\hline$C+Y_{L}$ & $12.83 \pm 0.44$ & $29.17 \pm 4.78^{*}$ & $1.62 \pm 0.03$ & $1.85 \pm 0.08^{*}$ \\
\hline$C+Y_{R}$ & $10.50 \pm 0.29 *$ & $17.83 \pm 1.42$ & $1.71 \pm 0.06$ & $1.77 \pm 0.07$ \\
\hline D & $11.00 \pm 3.5$ & $19.00 \pm 0.50 *$ & $1.42 \pm 0.08^{*}$ & $1.84 \pm 0.08^{*}$ \\
\hline$D+Y_{L}$ & $23.50 \pm 1.04^{* \#}$ & $25.83 \pm 1.17^{* \#}$ & $1.98 \pm 0.04^{* \#}$ & $1.99 \pm 0.04^{*}$ \\
\hline$D+Y_{R}$ & $16.50 \pm 1.19 * \#$ & $20.50 \pm 2.46^{*}$ & $1.52 \pm 0.04^{*}$ & $1.55 \pm 0.16$ \\
\hline
\end{tabular}

${ }^{*} \mathrm{P}<0.05$ compared with controls; ${ }^{\#} \mathrm{P}<0.05$ compared with diabetic rats; $\mathrm{Pl}_{30}$ - percentage of cells (phagocytic index) started the phagocytosis, of the total number of cells after 30 min of incubation; $\mathrm{Pl}_{120}$ - percentage of cells started the phagocytosis, of the total number of cells after 120 min of incubation; $\mathrm{PN}_{30}$ - average phagocytic number of yeasts cells that were inside phagocytes after 30 min of incubation; $\mathrm{PN}_{120}-$ average phagocytic number of yeasts cells that were inside phagocytes after 120 min of incubation; $C$ - control animals; $C+Y_{1}-$ control animals that were treated with extract of yacon leaves; $C+Y_{R}-$ control animals that were treated with extract of yacon root tubers; $D-$ animals with diabetes mellitus (DM); $D+Y_{L}-$ animals with DM that were treated with extract of yacon leaves; $D+Y_{R}-$ animals with $D M$ that were treated with extract of yacon root tubers

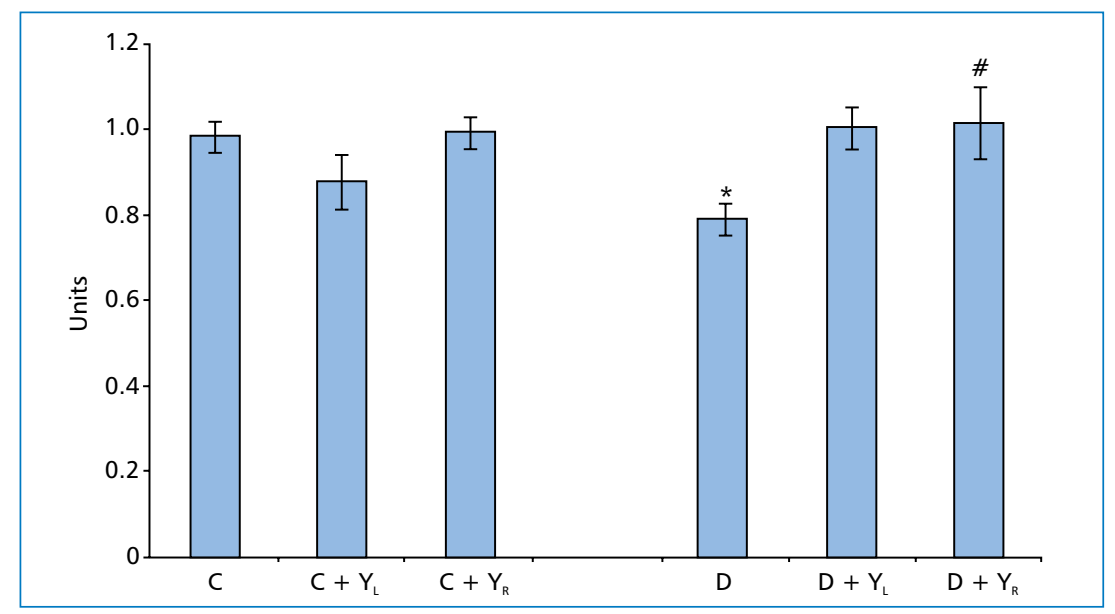

Figure 1. Changes in index of phagocytosis completeness under yacon extracts administration. ${ }^{*} \mathrm{P}<0.05$ compared with controls; ${ }^{\#} \mathrm{P}<0.05$ compared with diabetic rats; $\mathrm{C}-$ control animals; $\mathrm{C}+\mathrm{Y}_{\mathrm{L}}-$ control animals that were treated with extract of yacon leaves; $C+Y_{R}$ - control animals that were treated with extract of yacon root tubers; $D$ - animals with diabetes mellitus (DM); $D+Y_{L}$ - animals with DM that were treated with extract of yacon leaves; $D+Y_{R}$ - animals with DM that were treated with extract of yacon root tubers

cytosis process (120 minute), the amount of consumed yeast cells were reduced almost to control level (Table 1). Thus, the usage of $Y_{R}$ extract causes the growth of IPC to $28.5 \%$, comparing to DM (Figure 1 ).

In animals with experimentally induced diabetes MPO ACC increased by $16.4 \%$, compared to control animals. The use of $Y_{L}$ and $Y_{R}$ extracts caused no significant change in myeloperoxidase ACC in control animals. In DM reduction of ACC was established when using $Y_{L}$ extract (15.3\%) and when $Y_{R}$ extract (11.0\%) (Figure 2).

\section{Discussion}

The assessment of changes in phagocytic and bactericidal activity of PMN in diabetes and impact of agents with pronounced hypoglycemic effect on neutrophils functional activity is believed to be a promising research area. To test the phagocytic activity, rats' neutrophils were allowed to phagocytize unopsonized yeast cells. Changes in phagocytosis activity indexes (Table 1, Figure 1) indicate that neutrophils require more time for an adequate response to foreign cells and particles [5].

Considering that neutrophils generate low amounts of ATP in their mitochondria and thus rely primarily on glycolytic metabolism, altered glucose levels in diabetes impact on neutrophil function [9].

Abnormal leukocyte function in DM might be caused by the formation of an advanced glycation end products (AGEs). AGEs may cause aberrant signal processing with some serious consequences in terms of stimulus-response coupling, in particular leads to transient actin polymerization, which is required for chemotactic, phagocytic, and secretory responses. 


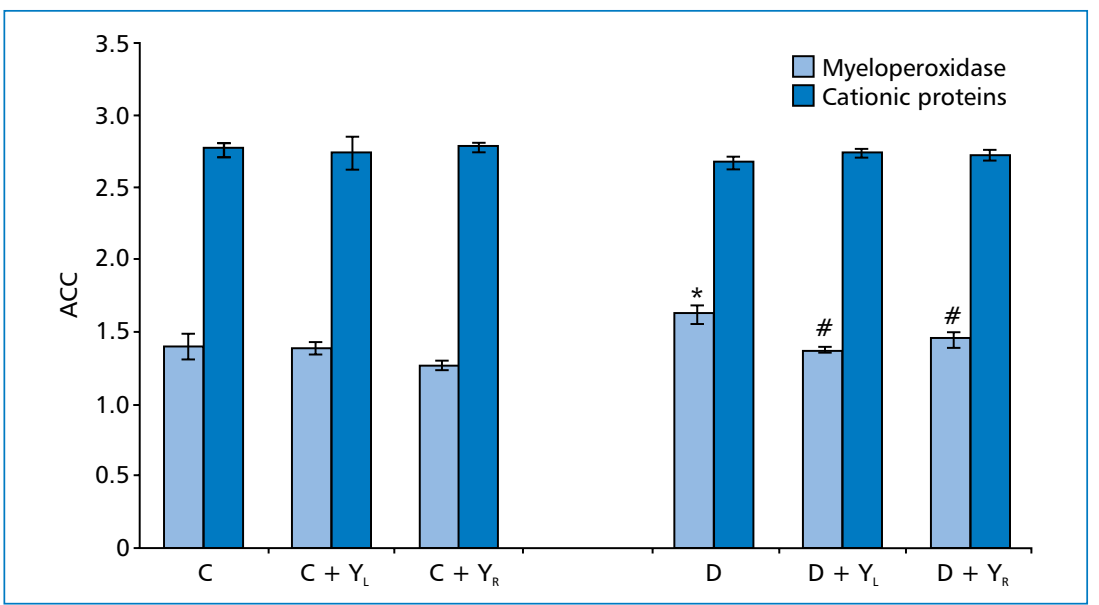

Figure 2. Changes in average cytochemical coefficient (ACC) of myeloperoxidase and cationic proteins under yacon extracts administration. ${ }^{*} \mathrm{P}<0.05$ compared with controls; ${ }^{*} \mathrm{P}<0.05$ compared with diabetic rats; $\mathrm{C}-$ control animals; $\mathrm{C}+\mathrm{Y}_{\mathrm{L}}-$ control animals that were treated with extract of yacon leaves; $C+Y_{R}-$ control animals that were treated with extract of yacon root tubers; $D$ - animals with diabetes mellitus (DM); $D+Y_{L}-$ animals with DM that were treated with extract of yacon leaves; $D+Y_{R}-$ animals with $D M$ that were treated with extract of yacon root tubers

The increased number of ingested yeast cells by neutrophils can also be associated with increased concentrations of AGEs. Products of nonenzymatic glycosylation cause the increase in the number of ingested bacteria per cell [10].

Intensification of phagocytosis process under $\mathrm{Y}_{\mathrm{L}}$ extract administration may be due to its pronounced hypoglycemic effect. This sugar lowering effect was shown in our previous research. Yacon leaves extracts in the condition of diabetes cause a decrease in glucose concentration by $13.4 \%$, while the root tubers extract caused a decrease in the content of the studied indicator by $49.9 \%$ [11]. Established effect may be due to the presence in yacon leaves sesquiterpene lactones, quercetin and other phenolic compounds, which have been shown to exhibit anti-inflammatory activity [12-14].

Demonstrated effect of $Y_{R}$ extract (Table 1, Figure 1) can be caused by high content in their composition of inulin type $\beta(2 \rightarrow 1)$ fructooligosaccharides (FOS) [13]. Short-term supplementation of FOS cause the increase in white blood cells phagocytic activity through binding to carbohydrate receptors $[4,15]$.

The phagocytic event stimulates the release of agents into the phagolysosome that will kill and digest the ingested microorganism. The non-oxygendependent mechanisms include the action of the enzymes contained in granules, such as antibacterial cationic proteins, lysozyme, various proteases, and direct effects of lactoferrin. The oxygen-dependent mechanisms are again of two types: MPO-dependent and MPO-independent. As a part of oxygen-dependent mechanisms of microorganism killing, we investigate the amount of MPO in PMN. Activated neutrophils release MPO from azurophilic granules at the sites of inflammation. MPO is able to interact with diverse ionic, atomic, and molecular entities via the interface with $\mathrm{H}_{2} \mathrm{O}_{2}$, including $\mathrm{HOCl}^{-}$, hydroxyl radicals, singlet oxygen, ozone, chloramines, and aldehydes. These species are potent oxidants, which under normal and controlled circumstances are toxic to several microorganisms and play an important role in the immune system [2].

The overproduction of MPO by neutrophils from animals with DM (Figure 2) can causes oxidative damage of proteins and DNA in host cells. Thus, the enhanced level of MPO is one of the best inflammatory and oxidative stress markers of diabetes [2]. This enzyme can bind other cell surfaces like epithelial cells, fibroblasts, endothelial cells, macrophages, platelets, neutrophils. The binding of enzyme to these cell surfaces alters some functional properties: interaction with neutrophil integrins causes enhanced tyrosine phosphorylation of some proteins. This activates protein tyrosine kinase, which results in degranulation and leads to respiratory burst. The binding of MPO to platelets causes the reorganization of platelet cytoskeleton, and thus alters the aggregation properties $[2,16]$.

Reduction of MPO ACC when using $Y_{L}$ leave extracts (Figure 2) can be caused by the presence in its composition phenolic antioxidants (ferulic acid, chlorogenic acid, gallic acid, caffeic acid, rosmarinic acid and quercetin) and flavonoids (apigenin and luteolin), which can inhibit MPO $[13,17,18]$. Somewhat less pronounced effect on MPO activity possessed $Y_{R}$ extract that may be due to lower content or lack of components that are capable to inhibit the activity of 




Figure 3. Generalizing scheme of yacon extracts influence on neutrophils functional activity under conditions of experimental diabetes mellitus (modified from Quinn et al. [20])

enzyme. FOS, which are the major biologically active substances in yacon root tubers also tended to reduce MPO activity [4].

In order to obtain additional data on the violation of bactericidal properties of neutrophils in diabetes, we investigate the amount of antibacterial cationic proteins as part of non-oxygen-dependent microbe killing mechanisms [19]. In our studies, we did not find significant change in cationic proteins ACC (Figure 2).

\section{Conclusions}

DM development was accompanied by reduced phagocytic activity of granulocytes with activation of oxygen-dependent mechanisms of microorganism killing. All used extracts intensified phagocytic activity of neutrophil in diabetes, with the yacon root tubers extract demonstrating the strongest effect, which was reflected by rapid degradation of particles engulfed by phagocytes. Yacon extracts possess inhibiting effect on myeloperoxidase activity in diabetes (Figure 3).

\section{Conflict of interests}

The authors declare that there is no conflict of interests regarding the publication of this paper.

\section{REFERENCES}

1. Hodgson $\mathrm{K}$, Morris J, Bridson $\mathrm{T}$, et al. Immunological mechanisms contributing to the double burden of diabetes and intracellular bacterial infections. Immunology. 2015; 144(2): 171-185, doi: 10.1111/imm.12394, indexed in Pubmed: 25262977.

2. Khan AA, Alsahli MA, Rahmani AH. Myeloperoxidase as an active disease biomarker: recent biochemical and pathological perspectives. Med Sci (Basel). 2018; 6(2), doi: 10.3390/medsci6020033, indexed in Pubmed: 29669993.

3. Yamaguchi R, Kawata J, Yamamoto T, et al. Mechanism of interferon-gamma production by monocytes stimulated with myeloperoxidase and neutrophil extracellular traps. Blood Cells Mol Dis. 2015; 55(2): 127-133, doi: 10.1016/j.bcmd.2015.05.012, indexed in Pubmed: 26142328.

4. Paredes LLR, Smiderle FR, Santana-Filho AP, et al. Yacon fructans (Smallanthus sonchifolius) extraction, characterization and activation of macrophages to phagocyte yeast cells. Int J Biol Macromol. 2018; 108: 1074-1081, doi: 10.1016/j.ijbiomac.2017.11.034, indexed in Pubmed: 29126938.

5. Nagalievska M, Sabadashka M, Hachkova H, et al. Galega officinalis extract regulate the diabetes mellitus related violations of proliferation, functions and apoptosis of leukocytes. BMC Complement Altern Med. 2018; 18(4): 1-13, doi: 10.1186/ /s12906-017-2079-3, indexed in Pubmed: 29310643.

6. Levinsky RJ, Harvey BA, Paleja S. A rapid objective method for measuring the yeast opsonisation activity of serum. J Immunol Methods. 1978; 24(3-4): 251-256, doi: 10.1016/00221759(78)90129-1, indexed in Pubmed: 363954.

7. Shubych MH. [Identification of cationic protein in the cytoplasm of leukocytes using bromophenol blue]. Tsytolohyia. 1974: 1321-1322.

8. Graham RC, Karnovsky MJ. The early stages of absorption of injected horseradish peroxidase in the proximal tubules of mouse kidney: ultrastructure cytochemistry by a new technique. J Histochem Cytochem. 1966; 14(4): 291-302.

9. Ratter JM, Tack C, Netea M, et al. Environmental signals influencing myeloid cell metabolism and function in diabetes. Trends Endocrinol Metab. 2018; 29(7): 468-480, doi: 10.1016/j. tem.2018.04.008.

10. Collison KS, Parhar RS, Saleh SS, et al. RAGE-mediated neutrophil dysfunction is evoked by advanced glycation end products (AGEs). J Leukoc Biol. 2002; 71(3): 433-444, indexed in Pubmed: 11867681.

11. Horbulinska O, Khokhla M, Hachkova G, et al. [Influence of yacon (Smallanthus sonchifolius Poepp. \& Endl.) on rats' blood cells the condition of experimental diabetes mellitus. Visnyk of Lviv University]. Biological Series. 2016; 71: 31-42.

12. Schorr K, Mefort I, Da Costa FB. A novel dimeric melampolide and further terpenoids from Smallanthus sonchifolius (Asteraceae) and the inhibition of the transcription factor NF- $\kappa$ B. Nat Prod Commun. 2007; 2: 367-374.

13. Lachman J, Fernandez EC, Orsak M. Yacon [Smallanthus sonchifolia (Poepp. et Endl.) H. Robinson] chemical composition and use - a review. Plant soil environ. 2003; 49(6): 283-90, doi: 10.17221/4126-PSE. 
14. Bub A, Watzl B, Blockhaus $M$, et al. Fruit juice consumption modulates antioxidative status, immune status and DNA damage. J Nutr Biochem. 2003; 14(2): 90-98, indexed in Pubmed: 12667600.

15. Vogt L, Ramasamy $U$, Meyer $D$, et al. Immune modulation by different types of $\beta 2 \rightarrow 1$-fructans is toll-like receptor dependent. PLoS One. 2013; 8(7): e68367, doi: 10.1371/journal.pone.0068367, indexed in Pubmed: 23861894.

16. Gorudko IV, Kostevich VA, Sokolov AV, et al. Functional activity of neutrophils in diabetes mellitus and coronary heart disease: role of myeloperoxidase in the development of oxidative stress. Bull Exp Biol Med. 2012; 154(1): 23-26, doi: 10.1007/s10517012-1865-7, indexed in Pubmed: 23330081.

17. Luan $H, K a n Z, X u Y$, et al. Rosmarinic acid protects against experimental diabetes with cerebral ischemia: relation to inflammation response. J Neuroinflammation. 2013; 10: 28, doi: 10.1186/17422094-10-28, indexed in Pubmed: 23414442

18. Zhang $\mathrm{Xu}$, Huang $\mathrm{H}$, Yang $\mathrm{T}$, et al. Chlorogenic acid protects mice against lipopolysaccharide-induced acute lung injury. Injury. 2010; 41(7): 746-752, doi: 10.1016/j.injury.2010.02.029, indexed in Pubmed: 20227691.

19. Ganz T. Defensins: antimicrobial peptides of innate immunity. Nat Rev Immunol. 2003; 3(9): 710-720, doi: 10.1038/nri1180, indexed in Pubmed: 12949495.

20. Quinn MT, Ammons MC, Deleo FR. The expanding role of NADPH oxidases in health and disease: no longer just agents of death and destruction. Clin Sci (Lond). 2006; 111(1): 1-20, doi: 10.1042/ CS20060059, indexed in Pubmed: 16764554. 